\title{
$\beta$-catenin mutation is correlated with a favorable prognosis in patients with hepatocellular carcinoma
}

\author{
ZHENG WANG $^{1,2^{*}}$, YUAN-YUAN SHENG ${ }^{2 *}$, XIAO-MEI GAO ${ }^{2}$, CHAO-QUN WANG $^{1,2}$, XIANG-YU WANG $^{1,2}$, \\ XU LU ${ }^{1,2}$, JIN-WANG WEI ${ }^{2}$, KAI-LI ZHANG ${ }^{2}$, QIONG-ZHU DONG ${ }^{1,2}$ and LUN-XIU QIN ${ }^{1,2}$ \\ ${ }^{1}$ Department of Surgery, Huashan Hospital, Fudan University, Shanghai 200040; \\ ${ }^{2}$ Institute of Biomedical Sciences, Fudan University, Shanghai 200032, P.R. China
}

Received December 8, 2014; Accepted April 15, 2015

DOI: $10.3892 / \operatorname{mco} .2015 .569$

\begin{abstract}
The $\beta$-catenin gene is frequently mutated in patients with hepatocellular carcinoma (HCC) and has long been thought to be one of the major oncogenes involved in the hepatocarcinogenesis. The prognostic role of $\beta$-catenin mutation in HCC remains unclear. To address this issue, a search for relevant studies was performed in the PubMed, Embase and Web of Science databases. The pooled effect was calculated from the available data to evaluate the correlation of $\beta$-catenin mutation with overall survival rate and tumor clinicopathological features in patients with HCC. The pooled odds ratio (OR) was calculated using the Mantel-Haenszel model for fixed effects. Three studies met the inclusion criteria. A total of 618 cases were included, and $\beta$-catenin mutation was identified in 104 of them. The meta-analysis revealed that the presence of $\beta$-catenin mutation $(n=104)$, compared with the control group $(\mathrm{n}=514)$, was correlated with a longer overall survival rate [OR, 0.33; 95\% confidence interval (CI), 0.21-0.53; $\mathrm{P}<0.00001]$ in patients with HCC. No significant heterogeneity was found among the eligible studies $\left(\mathrm{I}^{2}=0 \% ; \mathrm{P}=0.72\right)$. $\beta$-catenin mutation was correlated with a relatively lower rate of hepatitis $\mathrm{B}$ virus infection (OR, 0.36; 95\% CI, 0.21-0.61; $\mathrm{P}=0.0002)$, improved tumor differentiation (OR, 0.32; 95\% CI, 0.19-0.56; $\mathrm{P}<0.0001)$ and a lower tumor-node-metastasis stage (I+II) (OR, 0.23; 95\% CI, 0.14-0.38; $\mathrm{P}<0.00001)$. These findings suggest that $\beta$-catenin mutation may predict a favorable prognosis in patients with HCC.
\end{abstract}

Correspondence to: Professor Lun-Xiu Qin or Qiong-Zhu Dong, Department of Surgery, Huashan Hospital, Fudan University, 12 Middle Wulumuqi Road, Shanghai 200040, P.R. China

E-mail: qin_lx@yahoo.com

E-mail: qzhdong@fudan.edu.cn

${ }^{*}$ Contributed equally

Key words: hepatocellular carcinoma, $\beta$-catenin, mutation, prognosis, clinicopathological parameters, meta-analysis

\section{Introduction}

Hepatocellular carcinoma (HCC) is the sixth most common malignancy and the third most frequent cause of cancer-related fatality worldwide (1). It is estimated that 750,000 new cases occur globally per year, half of which are reported in China (2,3). Infection with hepatitis B virus (HBV) or hepatitis $\mathrm{C}$ virus (HCV) is thought to be the most frequent etiology of HCC (4). Additionally, interaction of aflatoxin B1 (AFB) exposure with HBV infection is believed to increase the prevalence of HCC (5). Tobacco, alcohol and obesity are also co-morbidity risk factors for $\operatorname{HCC}(6,7)$. However, environmental contributing factors can only partially explain the pathogenesis of HCC.

Hepatocarcinogenesis is a complex multistep process involving numerous signaling pathways (8). The signaling pathways, linked to distinct categories, such as proliferation, apoptosis, metabolism, splicing, chromatin biology and cell cycle, play crucial roles in the development of HCC (9). The Wnt- $\beta$-catenin signaling pathway regulates various processes that are important for cancer progression, including tumor initiation, growth, survival, migration, differentiation and apoptosis (10). Hyperactivation of this signaling pathway, stimulated by upstream molecules, occurs in a third of all HCCs and represents the major oncogenic driver (11).

$\beta$-catenin, the central hub of the canonical Wnt signaling pathway, has several biological functions that are referred to as oncogenic activities (12). Over the past few decades, numerous studies have explored the genetic profiles associated with cancer predisposition, and a high frequency of $\beta$-catenin mutation in HCC has been reported (13-17). According to the latest findings from whole-genome research in HCC patients, the $\beta$-catenin gene is the most frequently mutated oncogene (18). Additionally, several studies have shown that crosstalk between mutant $\beta$-catenin and other oncogenes or tumor suppressor genes, such as $H$-ras and Met, was involved in tumorigenesis in the liver $(19,20)$. In addition, it has been shown that a molecular subclass specific to $\beta$-catenin mutation in HCC was associated with certain clinical characteristics $(21,22)$. These previous studies indicate the importance of $\beta$-catenin mutation in the initiation and progression of HCC. However, limited evidence has indicated an association between $\beta$-catenin mutation and the survival rate in HCC 
patients, and the prognostic significance of $\beta$-catenin mutation in HCC patients remains unclear.

In the present study, a meta-analysis was performed of the published studies to investigate the prognostic significance of $\beta$-catenin mutation in HCC cases. The results showed that $\beta$-catenin mutation is correlated with favorable prognosis in HCC patients.

\section{Materials and methods}

Search strategy. Aa search of the PubMed, Embase and Web of Science databases was performed for the relevant studies published until September 1, 2014. The databases were searched using the following algorithm without language limits: (' $\beta$-catenin' OR 'beta-catenin' OR 'CTNNB1') and ('prognosis' or 'prognostic' or 'survival') and ('HCC' or 'hepatocellular neoplasm' or 'liver cancer' or 'HCC'). All the references cited in the relevant studies were used to identify additional eligible studies. For further full-text review and data extraction, only studies in English were included.

Inclusion and exclusion criteria. Studies were included in the present meta-analysis if they met the following inclusion criteria: i) Studies were written in the English language, ii) patients were diagnosed with $\mathrm{HCC}$ by pathological examination, iii) the samples used for the mutational analysis were obtained from surgical tumor tissue specimens, iv) $\beta$-catenin mutation was detected, and v) data were sufficient for the estimation of the hazard ratio (HR) or odds ratio (OR) and their respective $95 \%$ confidence intervals (CIs).

The following studies were excluded: i) Non-English publications; ii) reviews, letters, case reports and conference abstracts without original data; iii) duplications of previous studies and replicated samples; and iv) studies from which sufficient data could not be extracted.

Data extraction and quality assessment. Two investigators (Z Wang and YY Sheng) screened the included studies and independently extracted the raw data from all the eligible studies. For each study, the following information was collected: First author name, publication date, number of patients, gender of patients, tumor-node-metastasis stage, tumor differentiation, research technique, definition of $\beta$-catenin mutation and overall survival. Study quality was assessed independently by two investigators (Z. Wang and Y.Y. Sheng) according to the Newcastle-Ottawa scale (23). Disagreements were settled by discussion among the authors.

Statistical analysis. Outcomes of the eligible studies were analyzed in Review Manager Version 5.2. The statistical methods used were in accordance with the Cochrane Handbook for Systematic Review of Interventions (Version 5.1.0, updated March 2011).

The dichotomous variables were analyzed by estimating the OR and 95\% CI. The statistical method used was the Mantel-Haenszel fixed-effects model. The $\mathrm{I}^{2}$ value was calculated to evaluate the heterogeneity of the study, and heterogeneity was considered present if the $\mathrm{I}^{2}$ statistic was $\geq 50 \%$. All the P-values were found by a two-sided test and $\mathrm{P}<0.05$ was considered to indicate a statistically significant
699 studies identified after removal of duplicates

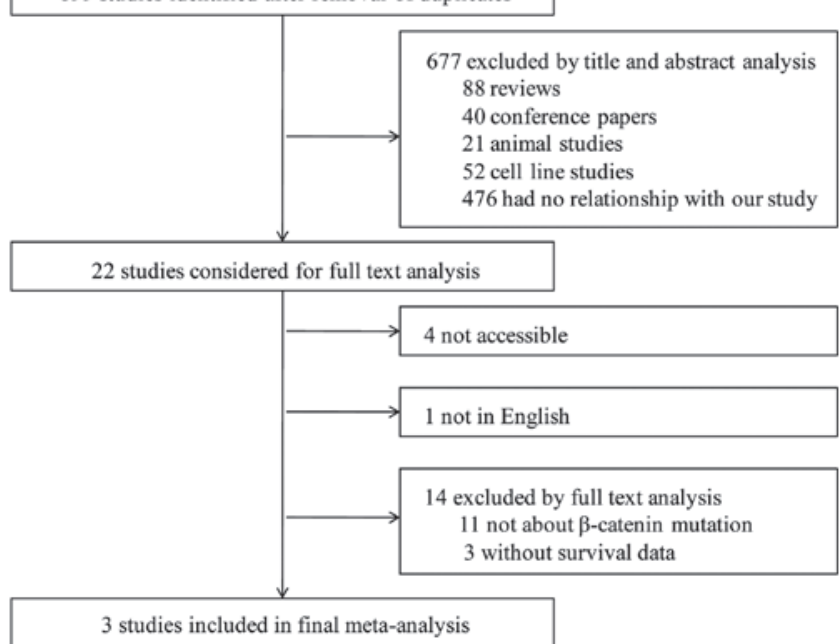

Figure 1. Flowchart of the selection process for the meta-analysis.

difference. A funnel plot was used to evaluate the potential publication bias.

\section{Results}

Description of studies. A flow diagram illustrating the search and selection criteria is provided in Fig. 1. The initial search strategy yielded 699 studies. On the basis of the titles and abstracts, 677 studies (including 88 reviews, 40 conference papers, 21 animal studies, 52 cell line studies and 476 studies that had no association with the present study) were excluded. Following screening of the full text, 4 studies were excluded that were unavailable, 1 was not written in English and 14 did not provide useful data. Three studies with a total number of 618 patients (104 with $\beta$-catenin mutations and 514 with wild-type $\beta$-catenin) were ultimately included in the analysis. Their characteristics are summarized in Table I. The studies in the meta-analysis were published between 2000 and 2011. Polymerase chain reaction (PCR) and DNA sequencing were used to detect $\beta$-catenin mutations. The association between $\beta$-catenin mutation and overall survival rate was investigated, and the end-point of follow-up was set at 5 years in all the studies.

Quality assessment. Each of the 3 studies eligible for analysis was assessed according to the 'Newcastle-Ottawa Scale (NOS) for assessing the quality of non-randomized studies in meta-analyses' standard. The NOS has 8 items categorized in three dimensions: Selection, comparability and exposure. A study can be awarded a maximum of 1 star for each numbered item within the selection and exposure categories, and a maximum of 2 stars in the comparability category. In total, quality scores range from 0 to 9 stars, and a study with a score of $\geq 6$ is defined as a high-quality study. On the basis of the NOS, all 3 studies included in the meta-analysis were of high quality (Table I).

Impact of $\beta$-catenin mutation on the overall survival rate in HCC patients. Among the included studies, the $\beta$-catenin 
Table I. Characteristics of the studies included in the meta-analysis.

First author Year Patients Comparison $\beta$-catenin mutation detection Mutation, \% Outcome measures Quality score ${ }^{\text {a }}$ (Refs.)

\begin{tabular}{|c|c|c|c|c|c|c|c|c|}
\hline $\mathrm{Hsu}$ & 2000 & 366 & Wild-type & DNA-sequencing & 12.8 & 5-year OS & 7 stars & $(25)$ \\
\hline Маo & 2001 & 66 & Wild-type & DNA-sequencing & 45.4 & 5-year OS & 6 stars & (27) \\
\hline Yuan & 2011 & 186 & Wild-type & DNA-sequencing & 14.5 & 5-year OS & 7 stars & (26) \\
\hline
\end{tabular}

'Quality of studies was assessed according to the 'Newcastle-Ottawa Scale for assessing the quality of non-randomized studies in meta-analyses' standard, and the number of stars was assigned.

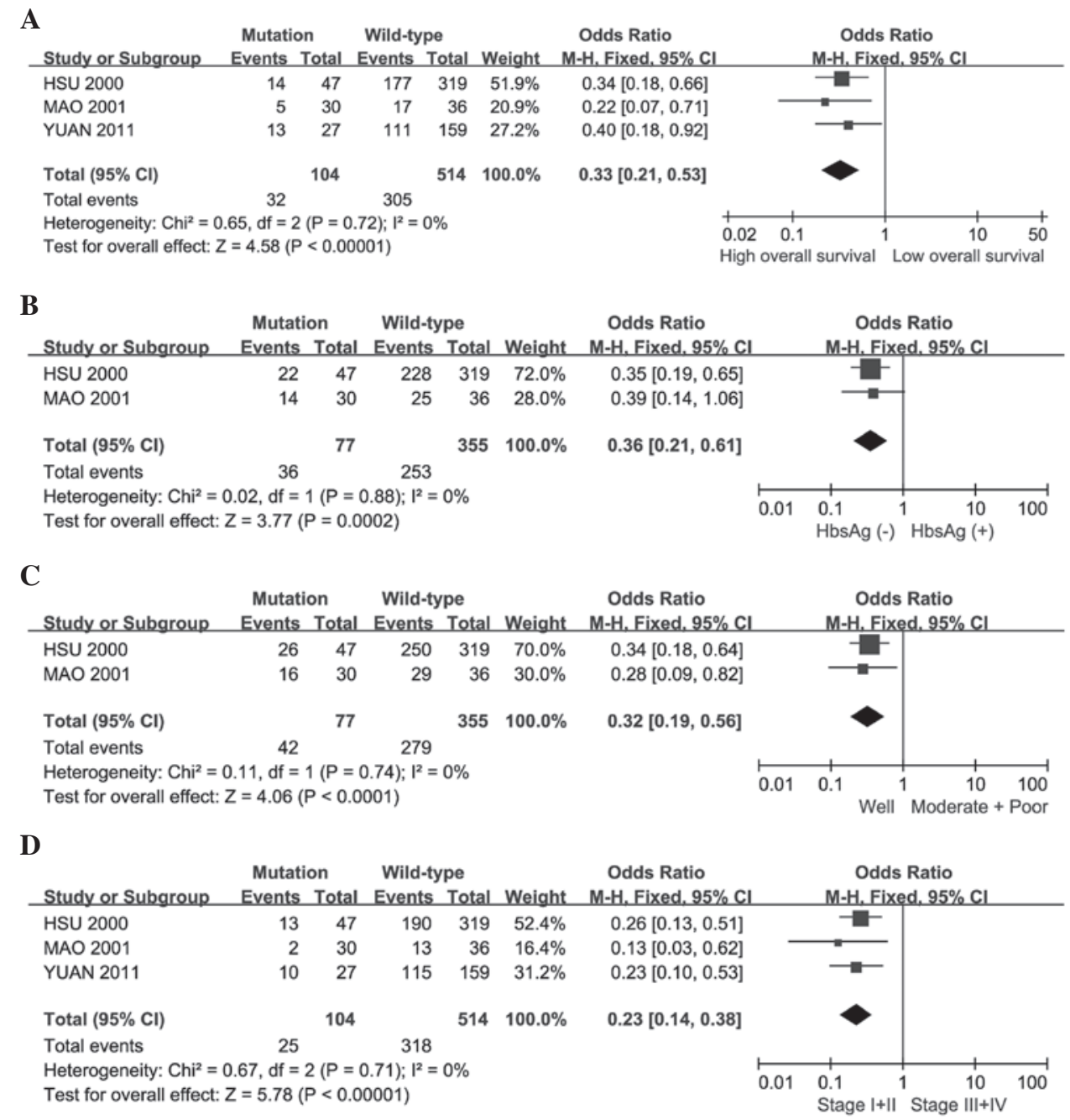

Figure 2. Forest plots. The results of the meta-analysis for (A) 5-year overall survival; (B) hepatitis B virus infection; (C) tumor differentiation; and (D) tumor-node-metastasis stage. CI, confidence interval.

mutation rate ranged from $12.8-44.4 \%$, and the mean incidence of $\beta$-catenin mutation was $16.6 \%$. The majority of mutations occurred at the phosphorylation sites for GSK-3 $\beta$ binding in exon 3 . The meta-analysis was conducted to assess the association between $\beta$-catenin mutation and the overall survival rate of $\mathrm{HCC}$ patients. The pooled OR was 0.33 (95\% CI, 0.21-0.53; Z=4.58; $\mathrm{P}<0.00001$ ) (Fig. 2A). The $\mathrm{I}^{2}$ test $\left(\mathrm{I}^{2}=0 \% ; \mathrm{P}=0.72\right)$ showed no significant heterogeneity between the 3 included studies. This result indicated that $\beta$-catenin mutation was correlated with a favorable prognosis in patients with HCC.
Correlation of $\beta$-catenin mutation with clinicopathological parameters. Two of the studies evaluated the correlation of $\beta$-catenin mutation with HBV infection. The pooled OR was 0.36 (95\% CI, 0.21-0.61; $\mathrm{Z}=3.77 ; \mathrm{P}=0.0002)$ (Fig. 2B) without heterogeneity $\left(\mathrm{I}^{2}=0 \% ; \mathrm{P}=0.88\right)$. This result indicated that $\beta$-catenin mutation occurred more frequently in patients who were negative for HBV. Additionally, 2 studies assessed the correlation between $\beta$-catenin mutation and tumor differentiation. The pooled OR was 0.32 (95\% CI, 0.19-0.56; $\mathrm{Z}=4.06$; $\mathrm{P}<0.0001$ ) (Fig. $2 \mathrm{C}$ ), suggesting that $\beta$-catenin mutation was associated with well-differentiated tumors. The 


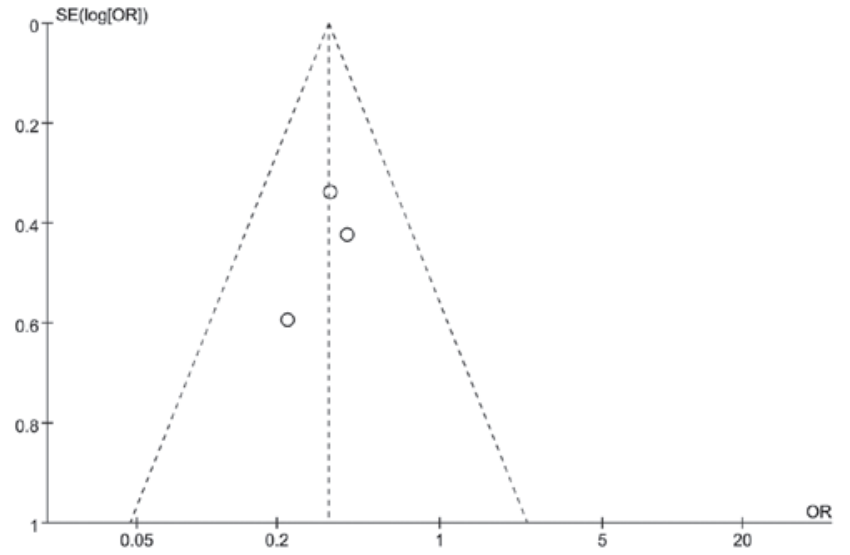

Figure 3. Funnel plot for the assessment of publication bias. SE, standard error; OR, odds ratio.

incidence of $\beta$-catenin mutation was significantly higher in stage I+II carcinomas compared to stage III+IV carcinomas (OR, 0.23; 95\% CI, 0.14-0.38; Z=5.78; P<0.00001) (Fig. 2D). Thus, $\beta$-catenin mutation was correlated with a subset of well-differentiated, low-stage HCCs in HBV-negative patients.

Publication bias. A funnel plot assessment was performed (Fig. 3), which indicated that no significant publication bias existed for the studies included in the meta-analysis.

\section{Discussion}

The Wnt signaling pathway plays a crucial role in the pathogenesis of HCC, and $\beta$-catenin serves as the core component of this pathway (24). The association between $\beta$-catenin mutation and prognosis in HCC patients was first reported in 2000 (25). Since then, however, few studies have further explored this area $(26,27)$. Therefore, it is necessary to analyze the existing data regarding $\beta$-catenin mutation in $\mathrm{HCCs}$ to draw a reasonable conclusion regarding its prognostic significance. The present systematic meta-analysis assessed comprehensive evidence regarding the prognostic significance of $\beta$-catenin mutation in HCC patients who received surgical treatment. The results suggest that the presence of $\beta$-catenin mutation predicts a favorable prognosis in HCC patients, which provides useful information for decision-making in the treatment of HCC.

$\beta$-catenin, a multifunctional cytoplasmic protein, is maintained at a low steady-state level in the absence of Wnt signaling. Alterations of the Wnt- $\beta$-catenin pathway are involved in the pathogenesis of HCC. $\beta$-catenin mutation, which is highly frequent in patients with HCC, can influence the proliferation, motility, apoptosis and senescence of cancer cells (28). It has been previously shown that $\beta$-catenin mutation was more frequent in small colorectal adenomas compared to larger adenomas and invasive carcinomas (29) and that it was associated with good clinical outcomes in ovarian carcinoma (30). These observations are consistent with the present finding that $\beta$-catenin mutation was significantly associated with a relatively good prognosis in HCC cases. Based on the findings of these previous studies, investigators have studied and identified possible mechanisms involved in this phenomenon. The studies by Sekine et al $(31,32)$ demonstrated that a $\beta$-catenin loss-of-function mutation affected glutamine synthesis pathway activity in mice and, thus, delayed hepatocyte proliferation following partial hepatectomy. Additionally, Apte et al (33) reported that $\beta$-catenin mutation, cooperating with the hepatocyte growth factor-mediated signaling pathway, impaired liver growth and minimized the pathogenesis of HCC. In addition, Harada et al (34) found that $\beta$-catenin gain-of-function mutations did not induce hepatocarcinogenesis in a mice model. These results may partially account for the correlation of $\beta$-catenin mutation with a longer survival rate in $\mathrm{HCC}$.

The present results also demonstrated that $\beta$-catenin mutation occurred more frequently in HBV-negative patients. This finding was supported by recent studies of whole-genome sequencing in $\mathrm{HCC}$, confirming that the rate of $\beta$-catenin mutation was much higher in HCV-related HCC compared to HBV-related HCC $(17,35,36)$. Additionally, the association between $\beta$-catenin mutation and clinicopathological features of HCC was analyzed and $\beta$-catenin mutation was associated with improved tumor differentiation and a lower tumor stage. The improved tumor differentiation and lower tumor stage may indicate a relatively lower primary tumor aggressiveness and a decreased probability of metastasis. This result is consistent with the present finding that $\beta$-catenin mutation predicts a favorable prognosis in HCC.

To avoid a potential influence of the studies with insufficient data on the reliability of this systematic review, only high-quality studies with large sample sizes $(\geq 100)$ were included in the meta-analysis, as these factors offer a more reliable analysis. In addition, all of the samples for the $\beta$-catenin mutation analysis were obtained from surgical tumor tissue specimens and the end-point of follow-up was set at 5 years in all the eligible studies. Furthermore, the combined analysis of the studies yielded similar results to those of the meta-analysis of all the eligible studies. This result demonstrated that the systematic review is propitious for the production and application of clinical evidence. As the participants in the meta-analysis were mostly from Asia, these findings may be more generally applicable to Asian patients. Therefore, to obtain a better understanding of the precise prognostic significance of $\beta$-catenin mutation, additional, large prospective studies with similar methods to those presented should be conducted.

Although the association between $\beta$-catenin mutation and patient prognosis in HCC was comprehensively evaluated, limitations exist in the present meta-analysis. First, the number of eligible studies included in the study was limited. Second, no unpublished studies were found. There was a possibility of publication bias, although the funnel plot showed no significant bias. Third, studies in languages other than English were excluded. The risk of language bias should be considered, but it may not lead to significant bias in the assessment of interventional effectiveness.

In conclusion, the present study indicated that the $\beta$-catenin mutation is correlated with a favorable prognosis and specific clinicopathological features in patients with HCC. As there is a paucity of evidence to clearly explain this finding, more scientific research is required to investigate the prognostic importance of 
$\beta$-catenin mutation among HCC patients, which may inform the choice of new adjunctive therapy for HCC cases.

\section{References}

1. Forner A, Llovet JM and Bruix J: Hepatocellular carcinoma. Lancet 379: 1245-1255, 2012.

2. Maluccio M and Covey A: Recent progress in understanding, diagnosing and treating hepatocellular carcinoma. CA Cancer J Clin 62: 394-399, 2012.

3. Jemal A, Bray F, Center MM, Ferlay J, Ward E and Forman D: Global cancer statistics. CA Cancer J Clin 61: 69-90, 2011.

4. Yang JD and Roberts LR: Hepatocellular carcinoma: A global view. Nat Rev Gastroenterol Hepatol 7: 448-458, 2010.

5. Ming L, Thorgeirsson SS, Gail MH, et al: Dominant role of hepatitis B virus and cofactor role of aflatoxin in hepatocarcinogenesis in Qidong, China. Hepatology 36: 1214-1220, 2002.

6. Marrero JA, Fontana RJ, Fu S, Conjeevaram HS, Su GL and Lok AS: Alcohol, tobacco and obesity are synergistic risk factors for hepatocellular carcinoma. J Hepatol 42: 218-224, 2005.

7. El-Serag HB: Hepatocellular carcinoma. N Engl J Med 365: 1118-1127, 2011.

8. Farazi PA and DePinho RA: Hepatocellular carcinoma pathogenesis: from genes to environment. Nat Rev Cancer 6: 674-687, 2006.

9. Garraway LA and Lander ES: Lessons from the cancer genome. Cell 153: 17-37, 2013.

10. Anastas JN and Moon RT: WNT signalling pathways as therapeutic targets in cancer. Nat Rev Cancer 13: 11-26, 2013.

11. Villanueva A, Newell P, Chiang DY, Friedman SL and Llovet JM: Genomics and signaling pathways in hepatocellular carcinoma. Semin Liver Dis 27: 55-76, 2007.

12. Grigoryan T, Wend P, Klaus A and Birchmeier W: Deciphering the function of canonical Wnt signals in development and disease: conditional loss- and gain-of-function mutations of beta-catenin in mice. Genes Dev 22: 2308-2341, 2008.

13. Miyoshi Y, Iwao K, Nagasawa Y, et al: Activation of the beta-catenin gene in primary hepatocellular carcinomas by somatic alterations involving exon 3. Cancer Res 58: 2524-2527, 1998.

14. Taniguchi K, Roberts LR, Aderca IN, et al: Mutational spectrum of beta-catenin, AXIN1 and AXIN2 in hepatocellular carcinomas and hepatoblastomas. Oncogene 21: 4863-4871, 2002.

15. Elmileik H, Paterson AC and Kew MC: Beta-catenin mutations and expression, 249serine p53 tumor suppressor gene mutation and hepatitis B virus infection in southern African Blacks with hepatocellular carcinoma. J Surg Oncol 91: 258-263, 2005.

16. Laurent-Puig P and Zucman-Rossi J: Genetics of hepatocellular tumors. Oncogene 25: 3778-3786, 2006

17. Fujimoto A, Totoki Y, Abe T, et al: Whole-genome sequencing of liver cancers identifies etiological influences on mutation patterns and recurrent mutations in chromatin regulators. Nat Genet 44: 760-764, 2012.

18. Kan Z, Zheng H, Liu X, et al: Whole-genome sequencing identifies recurrent mutations in hepatocellular carcinoma. Genome Res 23: 1422-1433, 2013.

19. Harada N, Oshima H, Katoh M, Tamai Y, Oshima M and Taketo MM: Hepatocarcinogenesis in mice with beta-catenin and Ha-ras gene mutations. Cancer Res 64: 48-54, 2004.

20. Tward AD, Jones KD, Yant S, et al: Distinct pathways of genomic progression to benign and malignant tumors of the liver. Proc Natl Acad Sci USA 104: 14771-14776, 2007.
21. Laurent-Puig P, Legoix P, Bluteau O, et al: Genetic alterations associated with hepatocellular carcinomas define distinct pathways of hepatocarcinogenesis. Gastroenterology 120: 1763-1773, 2001.

22. Hoshida Y, Nijman SM, Kobayashi M, et al: Integrative transcriptome analysis reveals common molecular subclasses of human hepatocellular carcinoma. Cancer Res 69: 7385-7392, 2009.

23. Stang A: Critical evaluation of the Newcastle-Ottawa scale for the assessment of the quality of nonrandomized studies in meta-analyses. Eur J Epidemiol 25: 603-605, 2010.

24. Gough NR: Focus issue: Wnt and beta-catenin signaling in development and disease. Sci Signal 5: eg2, 2012.

25. Hsu HC, Jeng YM, Mao TL, Chu JS, Lai PL and Peng SY: Beta-catenin mutations are associated with a subset of low-stage hepatocellular carcinoma negative for hepatitis B virus and with favorable prognosis. Am J Pathol 157: 763-770, 2000.

26. Yuan RH, Jeng YM, Hu RH, et al: Role of p53 and beta-catenin mutations in conjunction with CK19 expression on early tumor recurrence and prognosis of hepatocellular carcinoma. J Gastrointest Surg 15: 321-329, 2011.

27. Mao TL, Chu JS, Jeng YM, Lai PL and Hsu HC: Expression of mutant nuclear beta-catenin correlates with non-invasive hepatocellular carcinoma, absence of portal vein spread and good prognosis. J Pathol 193: 95-101, 2001.

28. Han ZG: Functional genomic studies: Insights into the pathogenesis of liver cancer. Annu Rev Genomics Hum Genet 13: 171-205, 2012.

29. Samowitz WS, Powers MD, Spirio LN, Nollet F, van Roy F and Slattery ML: Beta-catenin mutations are more frequent in small colorectal adenomas than in larger adenomas and invasive carcinomas. Cancer Res 59: 1442-1444, 1999.

30. Gamallo C, Palacios J, Moreno G, Calvo DMJ, Suarez A and Armas A: Beta-catenin expression pattern in stage I and II ovarian carcinomas: Relationship with beta-catenin gene mutations, clinicopathological features and clinical outcome. Am J Pathol 155: 527-536, 1999.

31. Sekine S, Lan BY, Bedolli M, Feng S and Hebrok M: Liver-specific loss of beta-catenin blocks glutamine synthesis pathway activity and cytochrome p450 expression in mice. Hepatology 43: 817-825, 2006.

32. Sekine S, Gutierrez PJ, Lan BY, Feng S and Hebrok M: Liver-specific loss of beta-catenin results in delayed hepatocyte proliferation after partial hepatectomy. Hepatology 45: 361-368, 2007.

33. Apte U, Zeng G, Muller P, et al: Activation of Wnt/beta-catenin pathway during hepatocyte growth factor-induced hepatomegaly in mice. Hepatology 44: 992-1002, 2006.

34. Harada N, Miyoshi H, Murai N, et al: Lack of tumorigenesis in the mouse liver after adenovirus-mediated expression of a dominant stable mutant of beta-catenin. Cancer Res 62: 1971-1977, 2002.

35. Guichard C, Amaddeo G, Imbeaud S, et al: Integrated analysis of somatic mutations and focal copy-number changes identifies key genes and pathways in hepatocellular carcinoma. Nat Genet 44: 694-698, 2012.

36. Li M, Zhao H, Zhang X, et al: Inactivating mutations of the chromatin remodeling gene ARID2 in hepatocellular carcinoma. Nat Genet 43: 828-829, 2011. 